\title{
List of Posters for Focus Meeting 20: Astronomy for Development
}

The following table contains a list of all posters and first authors that were registered to a participant during the the IAU General Assembly under Focus Meeting 20: Astronomy for Development. Due to the limited amount of time allocated for talks at the Focus Meeting, several Oral proposals had to be converted to Posters. We hope this table will be useful to illustrate the diversity of contributions to the Focus Meeting

Table 1: Titles and Authors for Poster Session at Focus Meeting 20: Astronomy for Development

\begin{tabular}{|c|c|}
\hline Titles & Author \\
\hline $\begin{array}{l}\text { Looking for Partners to Engage the Global } \\
\text { Community in Connecting to the Sky through } \\
\text { International Observe the Moon Night }\end{array}$ & $\begin{array}{l}\text { Sanlyn Buxner, Teaching, Learning, and } \\
\text { Sociocultural Studies, University of } \\
\text { Arizona, Tucson, Arizona, UNITED } \\
\text { STATES }\end{array}$ \\
\hline $\begin{array}{l}\text { Universe Awareness: a global educational } \\
\text { programme }\end{array}$ & $\begin{array}{l}\text { Tibisay Sankatsing Nava, Leiden } \\
\text { Observatory, Leiden University, Leiden, } \\
\text { NETHERLANDS }\end{array}$ \\
\hline How Big is Earth? & $\begin{array}{l}\text { Bonnie Thurber, Northwestern University, } \\
\text { Deerfield, Illinois, UNITED STATES }\end{array}$ \\
\hline $\begin{array}{l}\text { Astronomy and development: a multidisciplinary } \\
\text { project in the Mexican countryside }\end{array}$ & $\begin{array}{l}\text { Hector Bravo Alfaro, Departamento de } \\
\text { Astronomia, Universidad de Guanajuato, } \\
\text { Guanajuato, Guanajuato, MEXICO }\end{array}$ \\
\hline $\begin{array}{l}\text { Development Programs and Activities for } \\
\text { Southeast Asia Regional Office of Astronomy for } \\
\text { Development }\end{array}$ & \begin{tabular}{|l|} 
Wichan Insiri, Foreign Affairs \\
Department, National Astronomical \\
Research Institute of Thailand, \\
Chiangmai, THAILAND
\end{tabular} \\
\hline $\begin{array}{l}\text { Astronomy for Extremely ill or Traumatically } \\
\text { Injured Children and Their Families - IAU OAD } \\
\text { Grant }\end{array}$ & $\begin{array}{l}\text { Donald Lubowich, Physics and } \\
\text { Astronomy, Hofstra University, } \\
\text { Hempstead, New York, UNITED STATES }\end{array}$ \\
\hline $\begin{array}{l}\text { 3D Printing Meets Astrophysics: A New Way to } \\
\text { Visualize and Communicate Science }\end{array}$ & $\begin{array}{l}\text { Thomas Madura, NASA Goddard Space } \\
\text { Flight Center, Greenbelt, Maryland, } \\
\text { UNITED STATES }\end{array}$ \\
\hline Astronomy Outreach In Parana state/Brazil & $\begin{array}{l}\text { Marcelo Emilio, Geociências, Ponta } \\
\text { Grossa State University, Ponta Grossa, } \\
\text { Paraná, BRAZIL }\end{array}$ \\
\hline $\begin{array}{l}\text { "Astronomy for a Better World": IAU/OAD } \\
\text { Task Force One Activities to Develop Astronomy } \\
\text { Education and Research at Universities in the } \\
\text { Developing World }\end{array}$ & \begin{tabular}{|l|} 
Edward Guinan, Astrophysics \& \\
Planetary Science, Villanova University, \\
Villanova, Pennsylvania, UNITED \\
STATES
\end{tabular} \\
\hline $\begin{array}{l}\text { Current state of Czech astronomy popularization } \\
\text { and its potential for enhancing science career } \\
\text { interest }\end{array}$ & $\begin{array}{l}\text { Radek Kř́ícek, Astronomical Institute, } \\
\text { Charles University in Prague, Prague, } \\
\text { CZECH REPUBLIC }\end{array}$ \\
\hline
\end{tabular}


Table 1: Continued.

\begin{tabular}{|c|c|}
\hline Titles & Authors \\
\hline $\begin{array}{l}\text { Peer-review Platform for Astronomy Education } \\
\text { Activities }\end{array}$ & $\begin{array}{l}\text { Thilina Heenatigala, Leiden Observatory, } \\
\text { Leiden University, Leiden, } \\
\text { NETHERLANDS }\end{array}$ \\
\hline Astronomy in the DPR Korea & $\begin{array}{l}\text { George Miley, Leiden University, Leiden, } \\
\text { NETHERLANDS }\end{array}$ \\
\hline $\begin{array}{l}\text { Leiden University astronomy for development } \\
\text { projects }\end{array}$ & $\begin{array}{l}\text { George Miley, Leiden University, Leiden, } \\
\text { NETHERLANDS }\end{array}$ \\
\hline $\begin{array}{l}\text { A Pilot Astronomy Outreach Project in } \\
\text { Bangladesh }\end{array}$ & $\begin{array}{l}\text { Dipen Bhattacharya, Dept. of Natural } \\
\text { Sciences, Moreno Valley College, Moreno } \\
\text { Valley, California, UNITED STATES } \\
\end{array}$ \\
\hline $\begin{array}{l}\text { The Sharjah Center for Astronomy and Space } \\
\text { Sciences (SCASS 2015): Concept and Resources }\end{array}$ & $\begin{array}{l}\text { Hamid Al Naimiy, Office of the } \\
\text { Chancellor, University of Sharjah, } \\
\text { Sharjah, Sharjah, UNITED ARAB } \\
\text { EMIRATES }\end{array}$ \\
\hline $\begin{array}{l}\text { AstroNavigation: Freely-available Online } \\
\text { Instruction for Performing a Sight Reduction }\end{array}$ & \begin{tabular}{|l|} 
Susan Stewart, Astronomical \\
Applications, U.S. Naval Observatory, \\
Nashville, Tennessee, UNITED STATES
\end{tabular} \\
\hline $\begin{array}{l}\text { Principles of Celestial Navigation: An Online } \\
\text { Resource for Introducing Practical Astronomy to } \\
\text { the Public }\end{array}$ & \begin{tabular}{|l|} 
Sean Urban, US Naval Observatory, \\
Washington, District of Columbia, \\
UNITED STATES
\end{tabular} \\
\hline Journey of Ethiopia Astronomy & $\begin{array}{l}\text { Solomon Tessema, Astronomy and } \\
\text { Astrophysics Research Division, Entoto } \\
\text { Observatory and Research Center, Addis } \\
\text { Ababa, ETHIOPIA }\end{array}$ \\
\hline $\begin{array}{l}\text { Recent activities in Armenia related to IAU } \\
\text { ROAD and strategic plan }\end{array}$ & $\begin{array}{l}\text { Areg Mickaelian, Armenian Virtual } \\
\text { Observatory (ArVO), Byurakan } \\
\text { Astrophysical Observatory (BAO), } \\
\text { Yerevan, ARMENIA }\end{array}$ \\
\hline $\begin{array}{l}\text { Visiting nursery, kindergarten and after-school } \\
\text { day care as astronomy for development }\end{array}$ & $\begin{array}{l}\text { Akihiko Tomita, Wakayama University, } \\
\text { Wakayama, JAPAN }\end{array}$ \\
\hline $\begin{array}{l}\text { Design, transport, and installation of } \\
\text { autonomous Cherenkov detectors at high altitude }\end{array}$ & $\begin{array}{l}\text { Mario Calderón Cueva, Escuela } \\
\text { Politécnica Nacional, Quito, Pichincha, } \\
\text { ECUADOR }\end{array}$ \\
\hline $\begin{array}{l}\text { Results from pre- and post- tests of professional } \\
\text { development astronomy workshops for teachers } \\
\text { in Peru }\end{array}$ & $\begin{array}{l}\text { Susana Deustua, STSCI, Baltimore, } \\
\text { Maryland, UNITED STATES }\end{array}$ \\
\hline $\begin{array}{l}\text { ISODEX: An entry point for developing } \\
\text { countries into space activities }\end{array}$ & $\begin{array}{l}\text { Mark Skinner, Boering Research \& } \\
\text { Technology, The Boeing Company, } \\
\text { Albuquerque, New Mexico, UNITED } \\
\text { STATES }\end{array}$ \\
\hline $\begin{array}{l}\text { Exploring new possibilities of astronomy } \\
\text { education and outreach }\end{array}$ & $\begin{array}{l}\text { Kodai Fukushima, Hosei University, } \\
\text { Koganei, JAPAN }\end{array}$ \\
\hline eGTTP - bridging distances with online training & $\begin{array}{l}\text { Rosa Doran, Executive Council, NUCLIO } \\
\text { - Nucleo Interativo de Astronomia, Sao } \\
\text { Domingos de Rana, PORTUGAL }\end{array}$ \\
\hline $\begin{array}{l}\text { NASE 2015: Implementation of a Management } \\
\text { Quality System }\end{array}$ & $\begin{array}{l}\text { Rosa Ros, Applied Mathematics } 4, \\
\text { Technical University of Catalonia, } \\
\text { Barcelona, SPAIN }\end{array}$ \\
\hline
\end{tabular}

